\title{
Study on Image Recognition Application Based on Multi Features Fusion
}

\author{
Lelai DENG \\ Beijing Jiaotong University \\ Beijing City, Haidian District, 100044, China
}

\author{
Xiaoming HUANG \\ Beijing Jiaotong University \\ Beijing City, Haidian District, 100044, China
}

\begin{abstract}
From low to high, information fusion can be divided into three levels. The feature-level fusion not only keeps the most original information, but also overcomes the unstable and large characteristics of original data. In this article, we take six types of $B$ ultrasound liver images as the research objects, and with multi-feature fusion technology and its application in medical image recognition, made in-depth study on feature extraction of image color, texture and shape, and then apply them in multi-features fusion.
\end{abstract}

Keywords- Image Recognition; Multi Feature Fusion; Feature Extraction

\section{INTRODUCTION}

With the development of computer technology, Information fusion technology has become a new data processing technique. Information fusion technology has been phenomenal growth in the past 10 years, and entered many application fields. Image fusion is an important branch of information fusion technology, including three levels: pixel level, feature level and decision level. Its major application directions are shown in Figure 1:

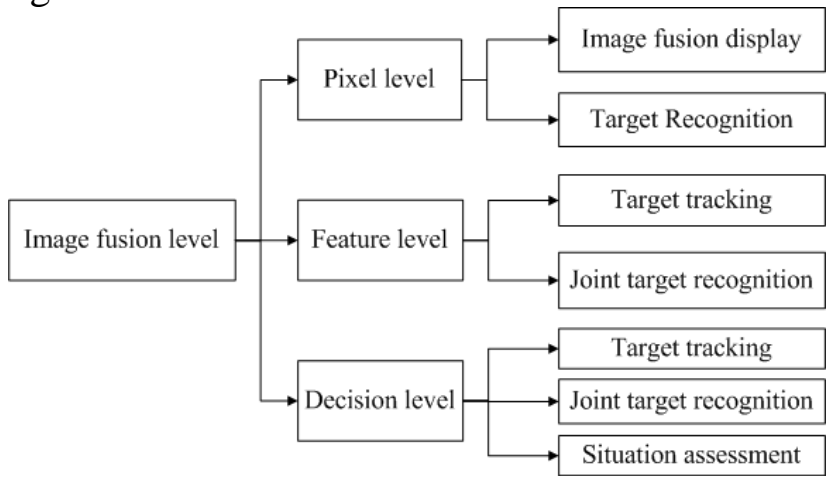

Figure 1. Application Fields of Image Fusion

Currently, image information fusion technology is widely used in military target recognition, medical diagnostics, remote sensing, computer vision, and weather forecasts, etc. Feature level image fusion can be divided into fusion target state information fusion and target feature fusion. Among them, target state information fusion is mainly used in the field of multi sensor target tracking, while target feature fusion is joint target recognition in feature level, which fusion method still involves pattern recognition technologies, but must carries on related processing of the feature before fusion. What's more important, in the field of pattern recognition, image processing and computer vision, people have do in-depth study on feature extraction and characteristics-based clustering problem. In fusion process, people often use fuzzy method, neural network technology, clustering method and Kalman filter, etc. The methods of fused image feature extraction can be divided into three categories: a) fusion of various image features in a single image; b) multi-image fusion of similar sensor in one scene; c) multi-image fusion of different sensing mode.

Medical image and common image is very different, due to its high gray resolution, large amount information, heterogeneity and noise significance characteristics. And Existing feature extraction methods still cannot well express the content of medical images. To solve this problem, in this article, we are committed to medical image feature extraction of color, texture and shape features, and based on application of principal component analysis (PCA) in feature level fusion, to analyze the liver B-ultrasound image recognition. 


\section{IMAGE FEATURE EXTRACTION}

\section{A. Image Acquisition}

The medical images we collected are 385 B ultrasound liver images, including six categories of hepatocellular carcinoma, hepatic cyst, liver hemangioma, fatty liver, hepatic congestion and normal liver. All the images are collected with the same ultrasound equipment, with JPEG format and $768 \times 57$ resolution. The six types of liver images are shown in Figure 2:

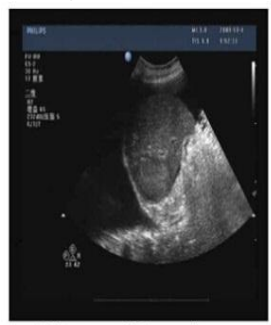

(a) hepatocellular carcinoma

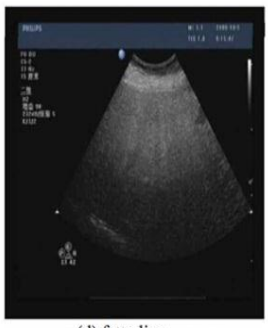

(d) fatty liver

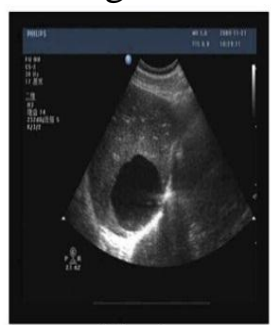

(b) hepatic cyst

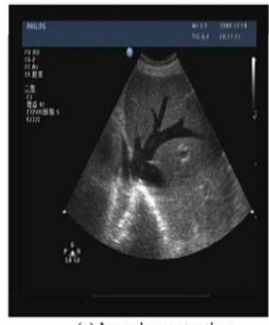

(e) hepatic congestion

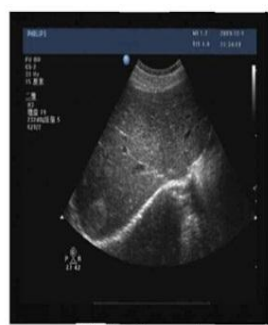

(c) liver hemangioma

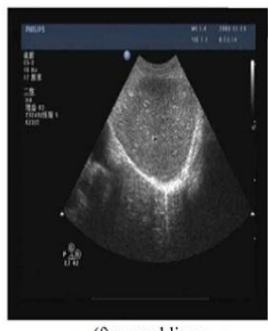

(f) normal liver
Figure 2. Six Types of Liver B-Ultrasonic Images

There are differences of different types of liver in Ultrasonic pulse reflection, Attenuation and absorption, so their B Ultrasonic images will have different performance in color, texture and shape, which reflects the pathology of the liver tissue.

\section{B. Feature Extraction}

\section{(1) Color feature}

The color feature of the image is visually intuitive, and its extraction has robust for any deformations of image translation, rotation, noise and even quality. The color moment method proposed by Stricker and Orengo considers that color information is concentrated in the low-end torque, and first moment (mean), second moment (variance) and third moment (slope) of each color component can effectively express the color distribution in an image. Color moments has the advantage of low character dimensions, and don't need color space quantization, so that can avoid the quantization effect on similarity discriminant.

Note $H\left(x_{i}\right)$ as Gray value of pixel $i$ in the image $X$, then its third-order color central moments are respectively as Formula (1) (3):

a) First moment:

错误!未找到引用 源。

b) Second moment:

错误!未找到引用

c) Third moment: 源。

错误!未找到引用 源。

The color moment features of six types of $B$ ultrasound images are shown in Table 1:

Table 1. Color Moment Features

\begin{tabular}{cccc}
\hline & First moment & Second moment & Third moment \\
\hline hepatocellular carcinoma & 50.7218 & 47.2718 & 55.1747 \\
hepatic cyst & 44.8219 & 43.8019 & 56.2819 \\
liver hemangioma & 46.0192 & 38.1238 & 45.1204 \\
fatty liver & 43.1910 & 31.4911 & 33.7757 \\
hepatic congestion & 53.9128 & 42.4226 & 46.2815 \\
normal liver & 54.4902 & 45.4122 & 51.8418 \\
\hline
\end{tabular}

From Table 1, we can see that in different moment, the color moment feature of normal liver and hepatocellular carcinoma is small; while there are significant differences between color moment feature of fatty liver and that of other types.

(2) Texture feature
Texture is an important feature to depict the space distribution of pixels' neighborhood gray in the image. It is generally defined as some local properties of the image, or a measure of the relationship between pixels in a local region.

We use gray level co-occurrence matrix (GLCM) method to accomplish texture feature 
extraction. GLCM not only contains gray statistic information, but also reflects the spatial information of gray distribution. Suppose the image has K gray levels, then the size of GLCM is $\mathrm{K} \times \mathrm{K}$. The space information refers to the frequency of a pair of pixels with certain distance and direction in GLCM. There are four typical directions: $\theta=0^{\circ}, 45^{\circ}, 90^{\circ}, 135^{\circ}$; common distance: $\mathrm{d}=1$ (for $\theta=0^{\circ}, 90^{\circ}$ ), or $\mathrm{d}=\sqrt{2}$ (for $\theta=45^{\circ}, 135^{\circ}$ ). For a $\mathrm{K} \times \mathrm{K}$ GLCM, its matrix element is equal to the quantity sum of all pixels that distance is $d$ and horizontal angle is $\theta$.

Defined elements of normalized GLCM C $(\mathrm{d}, \theta)$ as Formula (4):

\section{错误!未找到引用源。}

Size of GLCM depends on image total gray series. Haralick proposed 14 parameters based on normalized GLCM to describe the image texture feature. Among them, there are five typical parameters: angular second moment, inertia, inverse difference moment, entropy and correlation. Their calculation formula is shown as Formula (5) (9):

a) Angular second moment: 错误!未找到引 用源。

b) Inertia:

(6)

错误!未找到引用源。

c) Inverse difference moment: 错误!未找到 引用源。
d) Entropy:

(7)

(8)

$$
\text { e) Correlation: }
$$

错误!未找到引用源。

\section{源。9)}

We set distance $\mathrm{d}=1$ (for $\theta=0^{\circ}, 90^{\circ}$ ), $\mathrm{d}=\sqrt{2}$ (for $\theta=45^{\circ}, 135^{\circ}$ ). 错误!未找到引用源。 and $\mathrm{D}_{\mathrm{t}}$ is respectively mean and variance of the five basic parameters. Based on GLCM texture features, the value of the five parameters is shown in Table 2:

Table 2. Texture Features Based on GLCM

\begin{tabular}{ccccccc}
\hline & $\begin{array}{c}\text { Hepatocellular } \\
\text { carcinoma }\end{array}$ & $\begin{array}{c}\text { Hepatic } \\
\text { Cyst }\end{array}$ & $\begin{array}{c}\text { Liver } \\
\text { hemangioma }\end{array}$ & $\begin{array}{c}\text { Fatty } \\
\text { Liver }\end{array}$ & $\begin{array}{c}\text { Hepatic } \\
\text { congestion }\end{array}$ & Normal liver \\
\hline$\overline{\boldsymbol{t}_{\mathbf{1}}}$ & 30.4118 & 23.7818 & 25.9818 & 14.2648 & 25.8239 & 37.2199 \\
$\overline{\boldsymbol{t}_{\mathbf{2}}}$ & 1.28794 & 1.28599 & 1.30841 & 1.43910 & 1.25812 & 1.29581 \\
$\overline{\boldsymbol{t}_{\mathbf{3}}}$ & 4.02818 & 3.86221 & 4.20294 & 3.86471 & 4.17940 & 4.18284 \\
$\overline{\boldsymbol{t}_{\mathbf{4}}}$ & 0.00157482 & 0.00258521 & 0.0024845 & 0.00389491 & 0.00200194 & 0.00181024 \\
$\overline{\boldsymbol{t}_{\mathbf{5}}}$ & 0.148284 & 0.104859 & 0.091548 & 0.10284 & 0.080943 & 0.098592 \\
$\mathbf{D}_{\mathbf{t} \mathbf{1}}$ & 230.264 & 125.284 & 188.598 & 40.8591 & 172.093 & 289.488 \\
$\mathbf{D}_{\mathbf{t} \mathbf{2}}$ & 0.370952 & 0.369591 & 0.355271 & 0.400929 & 0.322841 & 0.318548 \\
$\mathbf{D}_{\mathbf{t 3}}$ & 2.00127 & 1.81048 & 2.18416 & 1.59827 & 2.23857 & 2.04719 \\
$\mathbf{D}_{\mathbf{t} 4}$ & $9.4892 \mathrm{e}-006$ & $2.5429 \mathrm{e}-005$ & $2.3152 \mathrm{e}-005$ & $5.0485 \mathrm{e}-005$ & $1.6284 \mathrm{e}-005$ & $1.1058 \mathrm{e}-005$ \\
$\mathbf{D}_{\mathbf{t}}$ & 0.502834 & 0.402585 & 0.395872 & 0.405883 & 0.408525 & 0.438582 \\
\hline
\end{tabular}

From Table 2, it is obvious that overall gray distribution of fatty liver is the most uniform, while that of hepatocellular carcinoma and normal liver is opponent; texture change of hepatic congestion is of regulation.

\section{(3) Shape feature}

Shape feature is one of the essential features of images, usually associated with the target of the image. The correspondingly characterization methods mainly include two categories: one is edge features, and another is regional features.

For digital image function $f(x, y)$, normalized second-order and third-order central moments can deduce seven invariant moments, as shown as Formula (10) (16):

错误!未找到引用源。

错误!未找到引用源。

错误!未找到引用源。

错误!未找到引用源。

错误!未找到引用源。 


\section{错误!未找到引用源。 错误!未找到引用源。}

Extract the invariant features of six types of liver B-Ultrasound images, as shown in Table 3:

Table 3. Invariant Moment Features

\begin{tabular}{ccccccc}
\hline & $\begin{array}{c}\text { Hepatocellular } \\
\text { carcinoma }\end{array}$ & $\begin{array}{c}\text { Hepatic } \\
\text { Cyst }\end{array}$ & $\begin{array}{c}\text { Liver } \\
\text { hemangioma }\end{array}$ & $\begin{array}{c}\text { Fatty } \\
\text { Liver }\end{array}$ & $\begin{array}{c}\text { Hepatic } \\
\text { congestion }\end{array}$ & Normal liver \\
\hline $\mathbf{T}_{\mathbf{1}}$ & 0.0134881 & 0.0150298 & 0.0131849 & 0.0136028 & 0.0140249 & 0.0098744 \\
$\mathbf{T}_{\mathbf{2}}$ & 0.00014859 & 000011858 & 0.00011084 & 0.00011357 & 0.000129428 & $7.5928 \mathrm{e}-005$ \\
$\mathbf{T}_{\mathbf{3}}$ & $2.00481 \mathrm{e}-006$ & $1.40284 \mathrm{e}-006$ & $1.10274 \mathrm{e}-006$ & $1.3028 \mathrm{e}-006$ & $1.5373 \mathrm{e}-006$ & $6.7824 \mathrm{e}-007$ \\
$\mathbf{T}_{\mathbf{4}}$ & $3.3728 \mathrm{e}-006$ & $3.4182 \mathrm{e}-006$ & $2.5623 \mathrm{e}-006$ & $3.0224 \mathrm{e}-006$ & $2.7942 \mathrm{e}-006$ & $1.3982 \mathrm{e}-006$ \\
$\mathbf{T}_{\mathbf{5}}$ & $8.9274 \mathrm{e}-012$ & $7.3573 \mathrm{e}-012$ & $3.9762 \mathrm{e}-012$ & $6.4313 \mathrm{e}-012$ & $5.9271 \mathrm{e}-012$ & $1.4022 \mathrm{e}-012$ \\
$\mathbf{T}_{\mathbf{6}}$ & $-1.6028 \mathrm{e}-009$ & $-6.92723 \mathrm{e}-009$ & $-2.9841 \mathrm{e}-009$ & $3.0945 \mathrm{e}-009$ & $-4.3942 \mathrm{e}-009$ & $-3.3940 \mathrm{e}-010$ \\
$\mathbf{T}_{\mathbf{7}}$ & $-8.8247 \mathrm{e}-012$ & $-4.2249 \mathrm{e}-012$ & $-3.6904 \mathrm{e}-012$ & $-6.744 \mathrm{e}-012$ & $-4.3928 \mathrm{e}-012$ & $-3.087 \mathrm{e}-010$ \\
$\boldsymbol{\theta}$ & 4.68724 & 0.37859 & -0.51724 & -0.19845 & -0.48957 & -3.48801 \\
$\boldsymbol{\varepsilon}$ & 0.73824 & 0.59847 & 0.65821 & 0.64829 & 0.68767 & 0.65838 \\
\hline
\end{tabular}

From Table 3, we can see that, the differences of moment features between normal liver and other liver images is significant, while the distributions of the different moments feature of every category change very little.

\section{CONCLUSIONS}

With the continuous development of computer technology, image processing and pattern recognition technology, feature-level image fusion technology is becoming a hot topic. However, currently its theoretical system and results evaluation system are not yet perfect, and most of the technical and theoretical methods are not universal.

In this article, we take six types of B ultrasound liver images as the research objects, and with multi-feature fusion technology and its application in medical image recognition, made in-depth study on feature extraction issues. Since a single feature is not well represent the medical image information, we research on the extraction method of medical images color, texture and shape features, by color moment features method, GLCM method and invariant moment feature method, an eventually apply them in feature fusion.

\section{Reference}

[1] Liu C, Wechsler H. A shape-and texture-based enhanced Fisher classifier for face recognition[J]. Image Processing, IEEE Transactions on, 2001, 10(4): 598-608.

[2] Chen J, He Y, Wang J. Multi-feature fusion based fast video flame detection[J]. Building and Environment, 2010, 45(5): 1113-1122.

[3] Sun Q S, Zeng S G, Liu Y, et al. A new method of feature fusion and its application in image recognition[J]. Pattern Recognition, 2005, 38(12): 2437-2448.

[4] ZHOU Y, Chen F, Sun B. Identification method of gas-liquid twophase flow regime based on image multi-feature fusion and support vector machine $[\mathrm{J}]$. Chinese Journal of Chemical Engineering, 2008, 16(6): 832-840.

[5] Pratt W K. Image feature extraction[J]. Digital Image Processing: PIKS Scientific Inside, Fourth Edition, 1978: 535-577.

[6] Hong Z Q. Algebraic feature extraction of image for recognition[J]. Pattern recognition, 1991, 24(3): 211-219.

[7] Lowe D G. Distinctive image features from scale-invariant keypoints[J]. International journal of computer vision, 2004, 60(2): 91-110. 